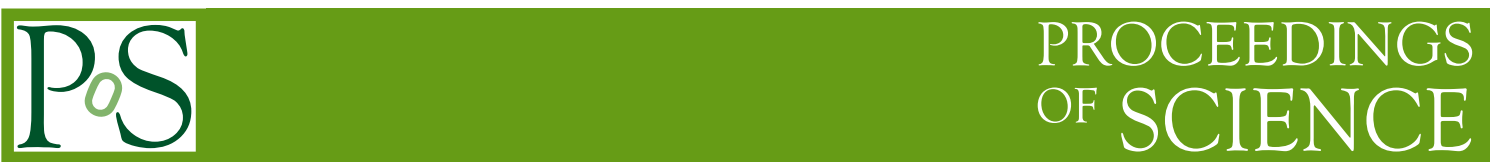

\title{
Multi-meson States in Lattice QCD
}

\section{William Detmold ${ }^{* \dagger}$}

Department of Physics, University of Washington, Seattle, WA 98195-1560, USA

E-mail: wdetmoldephys.washington.edu

In this contribution, I summarise the studies of the properties of Bose-Einstein condensed systems composed of up to twelve pions or kaons carried out by the NPLQCD collaboration. These investigations have provided precise determination the $\mathrm{I}=2 \pi \pi$ and $\mathrm{I}=1 K K$ scattering lengths and the first determination of three-hadron interactions fromm QCD, finding a repulsive threepion interaction of size consistent with naive dimensional analysis and a three kaon interaction consistent with zero. We have also determined the isospin (strangeness) density dependence of the isospin(strangeness) chemical potential, finding results in surprisingly good agreement with chiral perturbation theory.

The XXVI International Symposium on Lattice Field Theory

July 14-19 2008

Williamsburg, Virginia, USA

\footnotetext{
*Speaker.

†n behalf of the NPLQCD collaboration.
} 


\section{Many body lattice QCD}

Lattice QCD has had major impact in many aspects of particle physics phenomenology and in describing the spectra and structure of single hadrons. Computing resources and lattice algorithms have reached a stage where it is now worthwhile to consider the more complicated hadronic observables such as those in the baryon number, $B>1$ sector - the realm of nuclear physics. Here there are many observables that are phenomenologically important to nuclear structure and interactions and nuclear astrophysics about which very little (or nothing) is known experimentally or theoretically. Systems containing $n>2$ mesons are also of interest in a number of areas from RHIC to neutron stars. These systems present a significant opportunity for contributions from lattice QCD. Recently, the first attempts to study systems of more than two hadrons have been made by the NPLQCD collaboration [1, 2,3]. The results of these studies are summarised herein.

\section{Multi-meson systems}

It has long been known how to exploit the volume dependence of the eigen-energies of two hadron systems to extract infinite volume scattering phase shifts [4] provided that the effective range of the interaction, $r$ is small compared to the box size $L$ (since $r \sim m_{\pi}^{-1}$ for most interactions, this constraint is $m_{\pi} L \gg 1$ ). In recent works, this has been extended to systems involving $n>2$ bosons [5, 6, 7] and $n=3$ fermions [8] in the case when the relevant scattering length, $a$, is also small compared to the box size. The resulting shift in energy of $n$ particles of mass $M$ due to their interactions is

$$
\begin{aligned}
& \Delta E_{n}=\frac{4 \pi \bar{a}^{n}}{M L^{3}} C_{2}\left\{1-\left(\frac{\bar{a}}{\pi L}\right) \mathscr{I}+\left(\frac{\bar{a}}{\pi L}\right)^{2}\left[\mathscr{I}^{2}+(2 n-5) \mathscr{J}\right]\right. \\
& -\left(\frac{\bar{a}}{\pi L}\right)^{3}\left[\mathscr{I}^{3}+(2 n-7) \mathscr{I} \mathscr{J}+\left(5 n^{2}-41 n+63\right) \mathscr{K}\right] \\
& +\left(\frac{\bar{a}}{\pi L}\right)^{4}\left[\mathscr{I}^{4}-6 \mathscr{I}^{2} \mathscr{J}+\left(4+n-n^{2}\right) \mathscr{J}^{2}+4\left(27-15 n+n^{2}\right) \mathscr{I} \mathscr{K}\right. \\
& \left.\left.+\left(14 n^{3}-227 n^{2}+919 n-1043\right) \mathscr{L}\right]\right\} \\
& +{ }^{n} C_{3}\left[\frac{192 \bar{a}^{5}}{M \pi^{3} L^{7}}\left(\mathscr{T}_{0}+\mathscr{T}_{1} n\right)+\frac{6 \pi \bar{a}^{3}}{M^{3} L^{7}}(n+3) \mathscr{I}\right] \\
& +{ }^{n} C_{3} \frac{1}{L^{6}} \overline{\bar{\eta}}_{3}^{L}+\mathscr{O}\left(L^{-8}\right),
\end{aligned}
$$

where the parameter $\bar{a}$ is related to the scattering length, $a$, and the effective range, $r$, by

$$
a=\bar{a}-\frac{2 \pi}{L^{3}} \bar{a}^{3} r\left(1-\left(\frac{\bar{a}}{\pi L}\right) \mathscr{I}\right) .
$$

The geometric constants that enter into eq. (2.1) are

$$
\begin{array}{cc}
\mathscr{I}=-8.9136329, \mathscr{J}=16.532316, & \mathscr{K}=8.4019240, \\
\mathscr{L}=6.9458079, \mathscr{T}=-4116.2338, & \mathscr{T}_{1}=450.6392,
\end{array}
$$


and ${ }^{n} C_{m}=n ! / m ! /(n-m) !$. The three-body contribution to the energy-shift given in eq. (2.1) is represented by the parameter $\overline{\bar{\eta}}_{3}^{L}$, which is a combination of the volume-dependent, renormalization group invariant quantity, $\bar{\eta}_{3}^{L}$, and contributions from the two-body scattering length and effective range,

$$
\overline{\bar{\eta}}_{3}^{L}=\bar{\eta}_{3}^{L}\left(1-6\left(\frac{\bar{a}}{\pi L}\right) \mathscr{I}\right)+\frac{72 \pi \bar{a}^{4} r}{M L} \mathscr{I}
$$

where

$$
\bar{\eta}_{3}^{L}=\eta_{3}(\mu)+\frac{64 \pi a^{4}}{M}(3 \sqrt{3}-4 \pi) \log (\mu L)-\frac{96 a^{4}}{\pi^{2} M} \mathscr{S}_{\mathrm{MS}}
$$

The quantity $\eta_{3}(\mu)$ is the coefficient of the three- $\pi^{+}$interaction that appears in the effective Hamiltonian density describing the system [7]. It is renormalization scale, $\mu$, dependent. The quantity $\mathscr{S}$ is renormalization scheme dependent and we give its value in the minimal subtraction (MS) scheme, $\mathscr{S}_{\mathrm{MS}}=-185.12506$.

Lattice QCD measurements of these energy shifts allow one to extract the parameters $\bar{a}$ and $\overline{\bar{\eta}}_{3}^{L}$. To determine the energy shifts, we study the correlators (specifying to the multi-pion system)

$$
C_{n}(t) \propto\left\langle\left(\sum_{\mathbf{x}} \pi^{-}(\mathbf{x}, t)\right)^{n}\left(\pi^{+}(\mathbf{0}, 0)\right)^{n}\right\rangle,
$$

where $\pi^{+}(\mathbf{x}, t)=\bar{u}(\mathbf{x}, t) \gamma_{5} d(\mathbf{x}, t)$. On a lattice of infinite temporal extent, ${ }^{1}$ the combination

$$
G_{n}(t) \equiv \frac{C_{n}(t)}{\left[C_{1}(t)\right]^{n}} \stackrel{t \rightarrow \infty}{\longrightarrow} \mathscr{B}_{0}^{(n)} e^{-\Delta E_{n} t}
$$

where $\Delta E_{n}$ is the energy shift appearing in Eq. (2.1).

To compute the $(n !)^{2}$ Wick contractions in Eq. (2.4), we note that this correlation function can be written as

$$
C_{n}(t) \propto\left\langle(\bar{\eta} \Pi \eta)^{n}\right\rangle
$$

where

$$
\Pi=\sum_{\mathbf{x}} S(\mathbf{x}, t ; 0,0) S^{\dagger}(\mathbf{x}, t ; 0,0),
$$

and $S(\mathbf{x}, t ; 0,0)$ is a light-quark propagator. The object (block) $\Pi$ is a $12 \times 12$ (4-spin and 3 color) bosonic time-dependent matrix, and $\eta_{\alpha}$ is a twelve component Grassmann variable. Using

$$
\left\langle\bar{\eta}^{\alpha_{1}} \bar{\eta}^{\alpha_{2}} \ldots \bar{\eta}^{\alpha_{n}} \eta_{\beta_{1}} \eta_{\beta_{2}} \ldots \eta_{\beta_{n}}\right\rangle \propto \varepsilon^{\alpha_{1} \alpha_{2} . . \alpha_{n} \xi_{1} . \xi_{12-n}} \varepsilon_{\beta_{1} \beta_{2} . . \beta_{n} \xi_{1} . . \xi_{12-n}}
$$

leads to correlation functions

$$
C_{n}(t)=\varepsilon^{\alpha_{1} \alpha_{2} . \alpha_{n} \xi_{1} . . \xi_{12-n}} \varepsilon_{\beta_{1} \beta_{2} . . \beta_{n} \xi_{1} . . \xi_{12-n}}(\Pi)_{\alpha_{1}}^{\beta_{1}}(\Pi)_{\alpha_{2}}^{\beta_{2} . .}(\Pi)_{\alpha_{n}}^{\beta_{n}} .
$$

\footnotetext{
${ }^{1}$ Effects of temporal (anti-)periodicity are discussed in Ref. [3].
} 
For an arbitrary $12 \times 12$ matrix, $A$,

$$
\begin{aligned}
& \operatorname{det}(1+\lambda A)=\frac{1}{12 !} \varepsilon^{\alpha_{1} \alpha_{2} . \alpha_{12}} \varepsilon_{\beta_{1} \beta_{2} . . \beta_{12}}(1+\lambda A)_{\alpha_{1}}^{\beta_{1}}(1+\lambda A)_{\alpha_{2}}^{\beta_{2}} \ldots(1+\lambda A)_{\alpha_{12}}^{\beta_{12}} \\
& =\frac{1}{12 !}\left[\varepsilon^{\alpha_{1} \alpha_{2} . . \alpha_{12}} \varepsilon_{\alpha_{1} \alpha_{2} . . \alpha_{12}}+\lambda^{12} C_{1} \varepsilon^{\alpha_{1} \alpha_{2} . . \alpha_{12}} \varepsilon_{\beta_{1} \alpha_{2} . . \alpha_{12}}(A)_{\alpha_{1}}^{\beta_{1}}+\ldots\right. \\
& +\lambda^{n 12} C_{n} \varepsilon^{\alpha_{1} \alpha_{2} . . \alpha_{n} \xi_{1 . .} \xi_{12-n}} \varepsilon_{\beta_{1} \beta_{2} . . \beta_{n} \xi_{1} . \xi_{12-n}}(A)_{\alpha_{1}}^{\beta_{1}}(A)_{\alpha_{2}}^{\beta_{2}} \ldots(A)_{\alpha_{n}}^{\beta_{n}} \\
& \left.\ldots+\lambda^{12} \varepsilon^{\alpha_{1} \alpha_{2} . . \alpha_{12}} \varepsilon_{\beta_{1} \beta_{2} . . \beta_{12}}(A)_{\alpha_{1}}^{\beta_{1}} \ldots(A)_{\alpha_{12}}^{\beta_{12}}\right] \\
& =\frac{1}{12 !} \sum_{j=1}^{12}{ }^{12} C_{j} \lambda^{j} C_{j}(t)
\end{aligned}
$$

where in the last line we identify the matrix $A$ with $\Pi$. Further,

$$
\begin{aligned}
\operatorname{det}(1+\lambda A)= & \exp (\operatorname{Tr}[\log [1+\lambda A]])=\exp \left(\operatorname{Tr}\left[\sum_{p=1} \frac{(-)^{p-1}}{p} \lambda^{p} A^{p}\right]\right) \\
= & 1+\lambda \operatorname{Tr}[A]+\frac{\lambda^{2}}{2}\left((\operatorname{Tr}[A])^{2}-\operatorname{Tr}\left[A^{2}\right]\right) \\
& +\frac{\lambda^{3}}{6}\left(2 \operatorname{Tr}\left[A^{3}\right]-3 \operatorname{Tr}[A] \operatorname{Tr}\left[A^{2}\right]+(\operatorname{Tr}[A])^{3}\right)+\ldots
\end{aligned}
$$

Therefore, by equating terms of the same order in the expansion parameter $\lambda$ in Eq. (2.10) and Eq. (2.11), one can recover the $n-\pi^{+}$correlation functions in Eq. (2.9). As an example, the contractions for the $3-\pi^{+}$system are

$$
C_{3}(t) \propto \operatorname{tr}_{\mathrm{C}, \mathrm{S}}[\Pi]^{3}-3 \operatorname{tr}_{\mathrm{C}, \mathrm{S}}\left[\Pi^{2}\right] \operatorname{tr}_{\mathrm{C}, \mathrm{S}}[\Pi]+2 \operatorname{tr}_{\mathrm{C}, \mathrm{S}}\left[\Pi^{3}\right]
$$

where the traces, $\operatorname{tr}_{\mathrm{C}, \mathrm{S}}$, are over color and spin indices. Contractions for $n \leq 12$ mesons are given explicitly in Ref. [2].

\section{Two- and three- body interactions}

The NPLQCD collaboration have computed the $n$ pion and kaon correlators in the previous section using domain wall fermion [9, 10] propagators on various ensembles of MILC $2+1$ flavour rooted staggered gauge configurations [11] (parameters are shown in Table 1 and further details are given in Refs. [1, 2, 3]). In order to correctly calculate these correlators for large $n$, very high numerical precision is necessary (our calculations use the arprec library [12]). By performing a correlated fit to the effective energy differences extracted from these measurements, we have determined the two- and three-body interactions. The two body interactions extracted from this analysis agree with those extracted from the two-body sector alone [13]. The resulting three body interactions are displayed in Fig. 1. The three pion interaction is found to be repulsive with a magnitude consistent with the expectation from naive dimensional analysis. In contrast, the three $\mathrm{K}^{+}$interaction is consistent with zero within somewhat larger uncertainties. 
Table 1: The parameters of the MILC gauge configurations and domain-wall quark propagators used in these calculations. The subscript $l$ denotes light quark (up and down), and $s$ denotes the strange quark. The superscript $d w f$ denotes the bare-quark mass for the domain-wall fermion propagator calculation. The last column is the number of configurations times the number of sources per configuration. For the ensembles labeled with " $\mathrm{P} \pm \mathrm{A}$ ", propagators that were periodic in the temporal direction were computed in addition to those with anti-periodic temporal boundary conditions.

\begin{tabular}{cccccc}
\hline Ensemble & $b m_{l}$ & $b m_{s}$ & $b m_{l}^{d w f}$ & $b m_{s}^{d w f}$ & \# of propagators \\
\hline 2064f21b676m007m050 & 0.007 & 0.050 & 0.0081 & 0.081 & $1038 \times 24$ \\
2064f21b676m010m050 & 0.010 & 0.050 & 0.0138 & 0.081 & $768 \times 24$ \\
2064f21b679m020m050 & 0.020 & 0.050 & 0.0313 & 0.081 & $486 \times 24$ \\
2064f21b681m030m050 & 0.030 & 0.050 & 0.0478 & 0.081 & $564 \times 20$ \\
\hline 2896f2b709m0062m031 & 0.0062 & 0.031 & 0.0080 & 0.0423 & $1001 \times 7$ \\
2896f2b709m0062m031 $\mathrm{P} \pm \mathrm{A}$ & 0.0062 & 0.031 & 0.0080 & 0.0423 & $1001 \times(1+1)$ \\
\hline 2864f2b676m010m050 & 0.010 & 0.050 & 0.0138 & 0.081 & $137 \times 8$ \\
2864f2b676m010m050 $\pm \mathrm{A}$ & 0.010 & 0.050 & 0.0138 & 0.081 & $274 \times(2+2)$ \\
\hline
\end{tabular}

\section{Pion and kaon condensation}

The ground state of the $n$ meson systems that are being studied is a Bose-Einstein condensate of fixed $z$ component of isospin (and strangeness in the case of kaons). It is of great interest to investigate the properties of such systems. Theoretical efforts have used leading order chiral perturbation theory to investigate the phase diagram at low chemical potential [14] and it is important to assess the extent to which these results agree with QCD. Our numerical calculations allow us to probe the dependence of the energy on the pion (kaon) density, and thereby extract the chemical potential via a finite difference. The results using the coarse MILC lattice are shown for the pion and kaon systems in Figs. 2 and 3. Also shown is the prediction from tree-level chiral perturbation theory, with which we find surprisingly good agreement. This is encouraging for studies of kaon
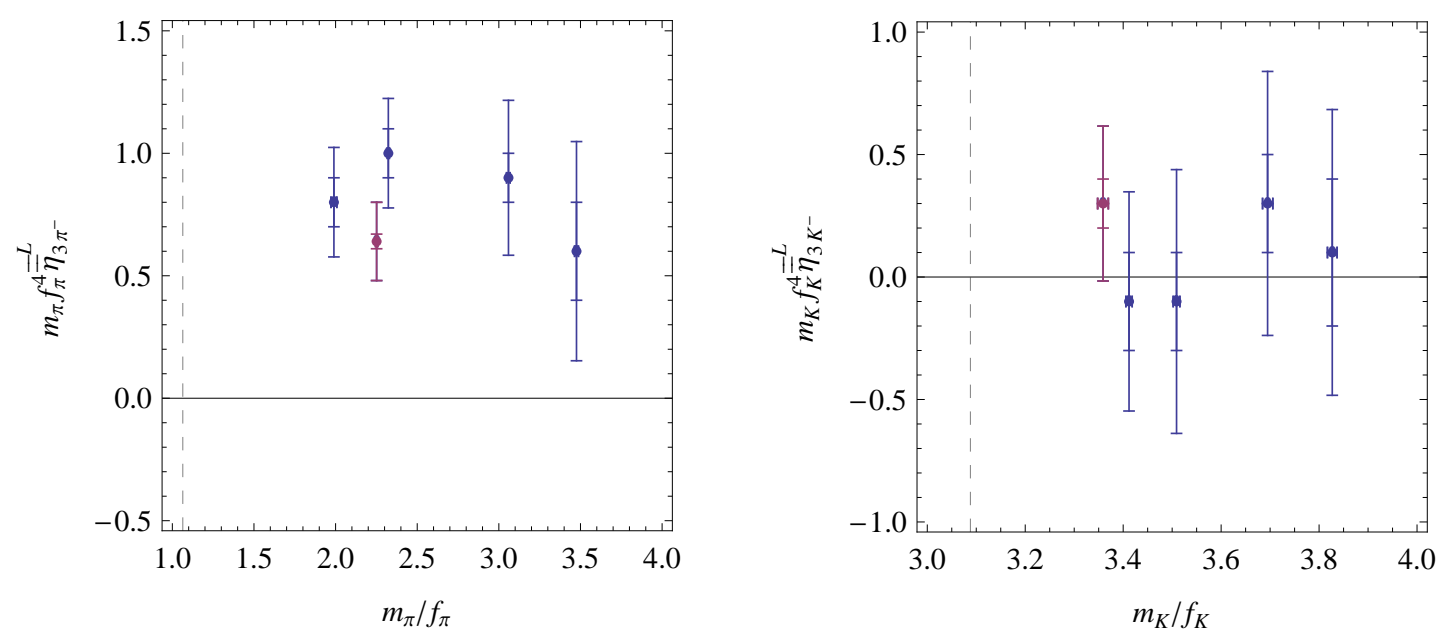

Figure 1: Three pion (left) and kaon (right) interactions determined from the MILC coarse (blue) and fine (magenta) lattices plotted . 

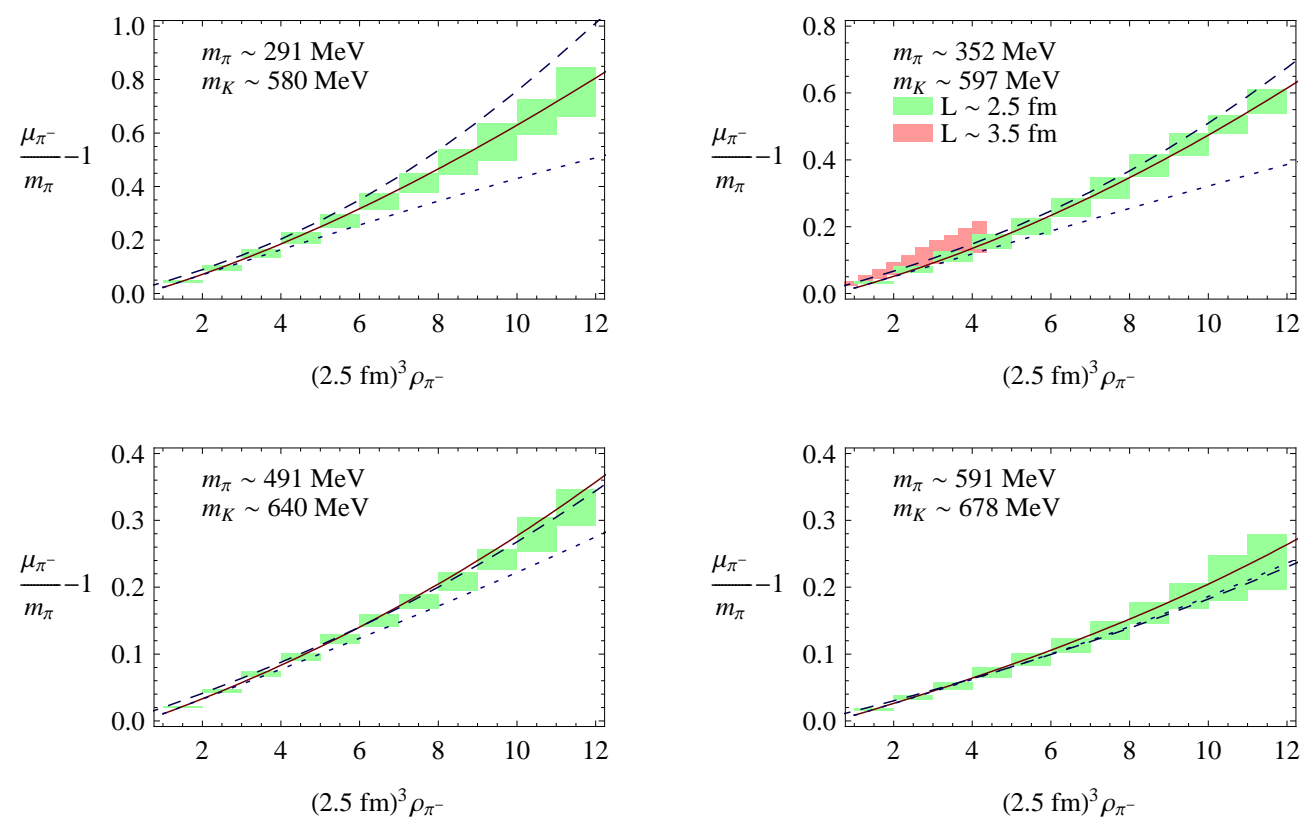

Figure 2: Dependence of the isospin chemical potential on the isospin density, calculated on the coarse MILC ensembles. The curves correspond to the predictions of tree level chiral perturbation theory (dashed) [14], the energy shift of Eq. (solid) and with the three-body interaction removed (dotted).

condensation in neutron stars where, typically, tree level chiral perturbation theory interactions are assumed amongst kaons and between kaons and baryons

\section{Summary}

Multi-meson systems (and in general multi-hadron systems) have been investigated using lattice QCD. The calculations presented here provide a first insight into the nature of these systems, but much remains to be studied. Recently, we have started to explore the effects of these condensed systems on other observables, looking at how the pion condensate screens the potential between a static quark-anti-quark pair [16].

\section{Acknowledgments}

We thank R. Edwards and B. Joo for help with the QDP++/Chroma programming environment [17].

\section{References}

[1] S. R. Beane, W. Detmold, T. C. Luu, K. Orginos, M. J. Savage and A. Torok, Phys. Rev. Lett. 100, 082004 (2008) [arXiv:0710.1827 [hep-lat]].

[2] W. Detmold, M. J. Savage, A. Torok, S. R. Beane, T. C. Luu, K. Orginos and A. Parreño, arXiv:0803.2728 [hep-lat] to appear in Phys. Rev. D. 

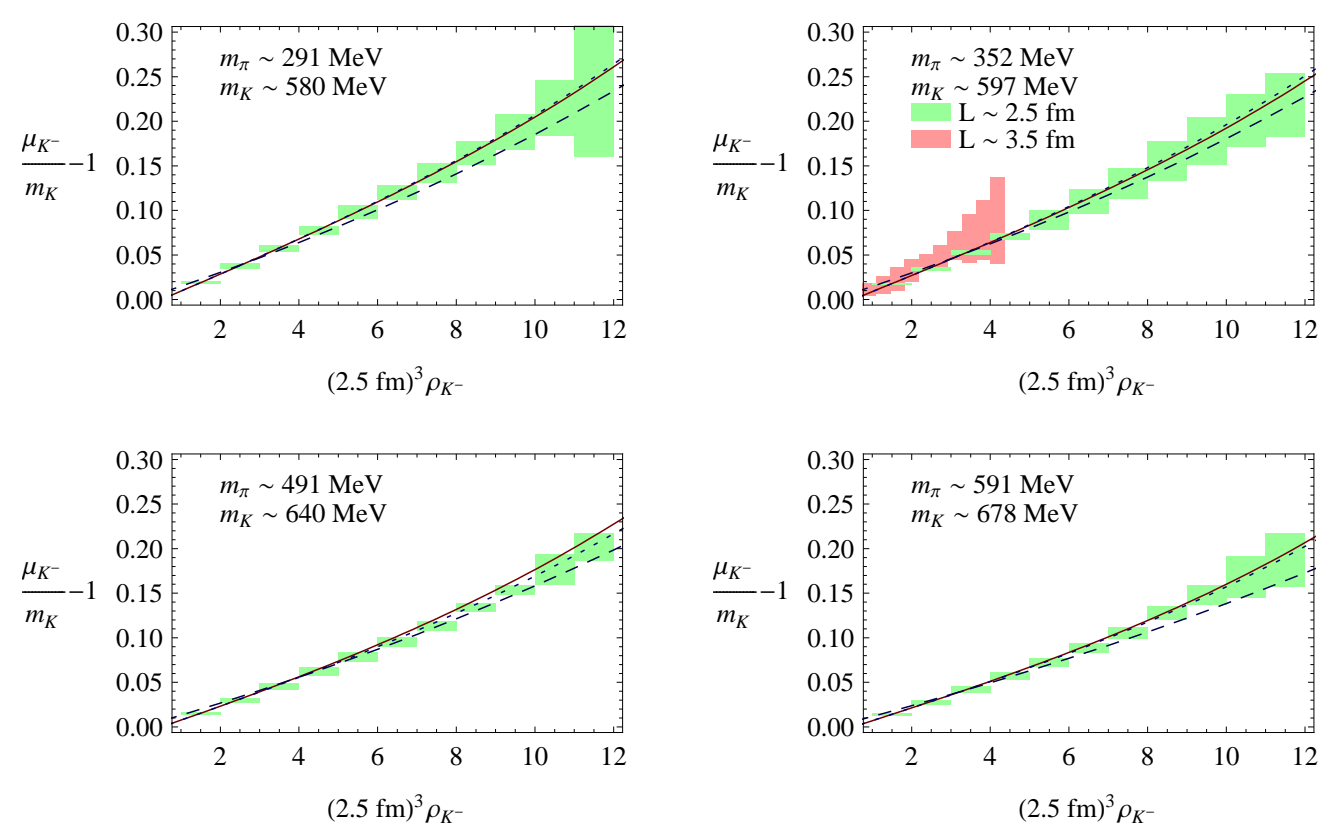

Figure 3: Dependence of the strangeness chemical potential on the kaon density. Details are as in Fig. 1.

[3] W. Detmold, K. Orginos, M. J. Savage and A. Walker-Loud, arXiv:0807.1856 [hep-lat].

[4] M. Lüscher, Commun. Math. Phys. 105, 153 (1986).

[5] S. R. Beane, W. Detmold and M. J. Savage, Phys. Rev. D 76, 074507 (2007) [arXiv:0707.1670 [hep-lat]].

[6] S. Tan, arXiv:0709.2530 [cond-mat.stat-mech].

[7] W. Detmold and M. J. Savage, Phys. Rev. D 77, 057502 (2008) [arXiv:0801.0763 [hep-lat]].

[8] T. Luu, "Three fermions in a box", these proceedings.

[9] D. B. Kaplan, Phys. Lett. B 288, 342 (1992) [arXiv:hep-lat/9206013].

[10] Y. Shamir, Nucl. Phys. B 406, 90 (1993) [arXiv:hep-lat/9303005].

[11] C. W. Bernard et al., Phys. Rev. D 64, 054506 (2001).

[12] David H. Bailey, Yozo Hida, Xiaoye S. Li and Brandon Thompson, "ARPREC: An Arbitrary Precision Computation Package," manuscript, Sept 2002; LBNL-53651. Available from http://crd.lbl.gov/ dhbailey/mpdist/.

[13] S. R. Beane, T. C. Luu, K. Orginos, A. Parreno, M. J. Savage, A. Torok and A. Walker-Loud, Phys. Rev. D 77, 014505 (2008) [arXiv:0706.3026 [hep-lat]].

[14] D. T. Son and M. A. Stephanov, Phys. Rev. Lett. 86, 592 (2001) [arXiv:hep-ph/0005225].

[15] D. B. Kaplan and A. E. Nelson, preprint HUTP-86/A023; Phys. Lett. B 175 (1986) 57.

[16] W. Detmold and M. J. Savage, arXiv:0809.0892 [hep-lat].

[17] R. G. Edwards and B. Joo [SciDAC Collaboration], Nucl. Phys. Proc. Suppl. 140 (2005) 832 [arXiv:hep-lat/0409003]. 